\title{
Seismic Characterization of an Abandoned Mine Site
}

\author{
Diego AROSIO ${ }^{1}$, Laura LONGONI ${ }^{2}$, Monica PAPINI ${ }^{2}$, and Luigi ZANZI ${ }^{1}$ \\ ${ }^{1}$ Department of Structural Engineering, Politecnico di Milano, Milan, Italy \\ e-mails: diego.arosio@polimi.it (corresponding author); luigi.zanzi@polimi.it \\ ${ }^{2}$ Department of Environmental, Hydraulic, Infrastructures \\ and Surveying Engineering, Politecnico di Milano, Milan, Italy \\ e-mails: laura.longoni@polimi.it; monica.papini@polimi.it
}

\begin{abstract}
The near-surface rock structure that covers an abandoned marl mine nearby the village of Montevecchia (Italy) was investigated through a combination of seismic surveys. The methods selected for these investigations were refraction seismics and multichannel analysis of surface waves (MASW). A sort of transillumination experiment was also attempted. All the methods were successful and gave complementary information. Refraction seismics was used to characterize the upper low velocity layer and the second layer of the near-surface structure. The MASW method was necessary to assess the existence of a velocity inversion revealing the presence of a low velocity layer between the 2 nd layer and a 4th high velocity layer covering the upper mine gallery. The transillumination experiment validated the presence of the 4th layer and gave an estimate of the average velocity that represents a lower boundary for the $P$-wave velocity within this layer. Both the refraction and transillumination data were analysed to derive average estimates of attenuation level and rock quality factor.
\end{abstract}

Key words: refraction seismics, MASW, abandoned mine.

\section{INTRODUCTION}

Montevecchia is an old historical village settled on the flanks of a $500 \mathrm{~m}$ high isolated hill located north of Milan (Northern Italy; Fig. 1). The hill was 
exploited in the 20th century to extract marl rock that was used to produce concrete. In 1958 a large sinkhole occurred on top of the hill because of a collapse involving all mine levels. Excavation works ceased, the mine was abandoned and the road running above the mine along the hill ridge had to be deviated and repaired. A new parking lot that still exists was also created in the sinkhole area (Fig. 1). Since then, no other maintenance works were done in the abandoned mine. Recently, an investigation program was planned by the regional authority to assess the safety conditions of the mine and the possible risk of other events like the 1958 sinkhole. Excluding direct inspections of the mine galleries for safety reasons (the upper gallery is unsafe and probably obstructed by the 1958 collapse, while all the other levels are flooded by water) all the geological and geophysical investigations had to be performed outside the mine except of a few measurements that could be taken at the entrance of the mine where the upper gallery departs from the main room of the mine.

Historical data analysis permitted to gather precious information concerning geological hazards affecting the mine. Collected data were evaluated to gain an understanding of the geological setting of the mine (i.e., features of subsurface layers), the behavior of mine during excavation and significant changes in groundwater circulation (i.e., springs disappearance). Classical geological mapping was performed and field data were analyzed in order to better understand rock mass state of fracturation and gather useful information to design a proper subsurface investigation program. The present work describes the geophysical investigations. The specific objective of the geophysical surveys was to assess the quality of the rock layers that cover the upper mine gallery. It is a rock structure, approximately $50 \mathrm{~m}$ thick, with a lateral extent of about $300 \mathrm{~m}$. We discuss the reasons that led us to design a combination of seismic experiments to accomplish the task. Subsequently, a short description of the acquisitions with a few examples of raw data images is presented and afterwards we separately describe the data processing and the interpretation of the results concerning each experiment. Finally, we discuss the complementary information derived by the methods and we draw the conclusions.

\section{DESIGN OF SEISMIC EXPERIMENTS}

Seismics is definitely the most interesting method among the geophysical techniques when the objective is the assessment of the rock mechanical properties. After a preliminary on-site inspection, we selected three techniques that were promising for the specific case: refraction seismics, multichannel analysis of surface waves (MASW), and transillumination experiments (a sort of tomography with very limited angular coverage because of 
the restrictions in entering the mine). Reflection seismics was excluded for the expected difficulties as well as for economic reasons. Considering that the objective is to characterize the properties of a near-surface rock structure that is only $50 \mathrm{~m}$ thick, a reflection experiment would be extremely demanding in terms of resolution.

Refraction seismics was preferred and a spread of 24 geophones with a spacing of $5 \mathrm{~m}$ was designed. The spread length results in $115 \mathrm{~m}$ and represents a good tradeoff between the need of long distances to observe headwaves from deep layers and the need of short distances to ensure a signal-tonoise-ratio (SNR) value high enough to allow a reliable first arrival picking. The whole road segment under investigation (Fig. 1) is about $300 \mathrm{~m}$ long so that three spreads were planned with partial superimposition (two geophones). Two seismic sources were considered as potentially suitable: an $8 \mathrm{~kg}$ hammer and a seismic gun. The final decision between the two options was deferred after the preliminary on-site test. Vertical low frequency geophones $(4.5 \mathrm{~Hz})$ were selected for the experiments so that refraction shots could be also analyzed with the MASW method that requires low frequencies to extend the $S$-wave model to greater depths.

The MASW method was considered for three reasons: (i) it is complementary to refraction seismics because it gives the $S$-wave model that combined with the $P$-wave model derived from the refraction method represents

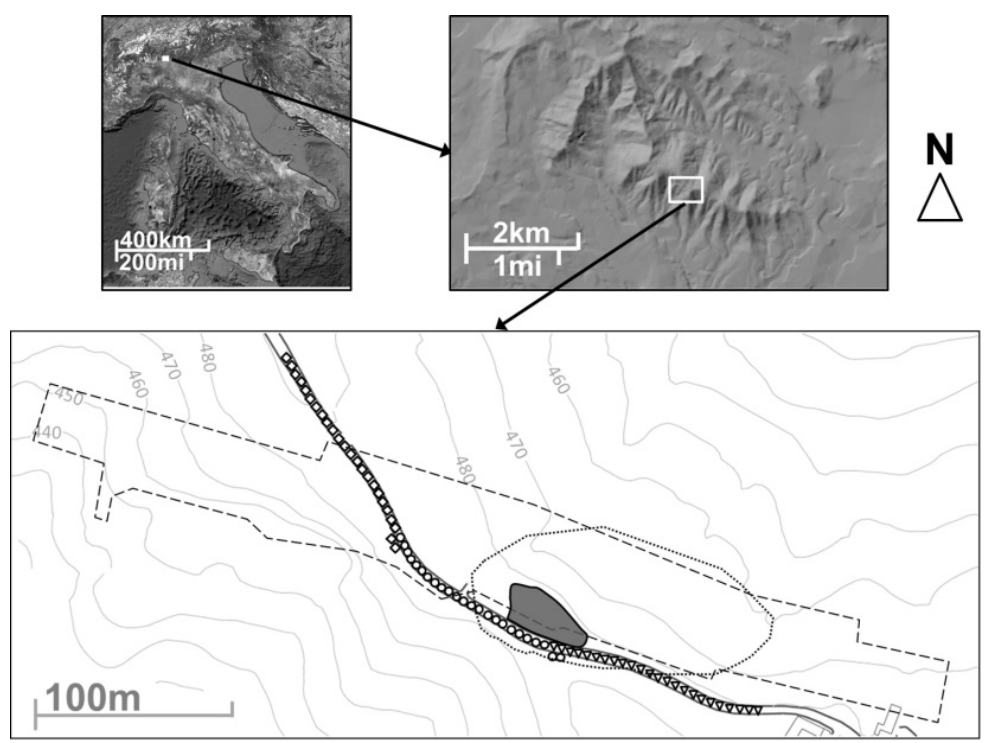

Fig. 1. Location of the investigated area. In the bottom image: dashed area is the contour of the mine footprint, dotted area delimits the 1958 sinkhole, shaded area is the parking lot; triangles, circles, and rhombs represent the three seismic spreads. 
a more complete picture of the elastic properties of the rock, (ii) it has the potential to detect velocity inversions that the refraction method cannot solve, and (iii) it increases the chances to reconstruct a velocity model down to the level of the upper gallery (the penetration of the refraction experiment is hardly predictable and might be prevented by velocity inversions).

Since the objective of the investigations is the rock structure above the mine, the ideal experiment for exploring the seismic velocity distribution would be a seismic tomography between the upper mine gallery and the road running above the mine. This experiment was not feasible for safety reasons but at least a transillumination test was planned taking advantage of the fact that the entrance of the upper gallery could be reached with limited risk. A few geophones at the entrance of the uppermost gallery and some shots distributed along the road that intersects the mine are expected to give an average estimate of the velocity of the $P$-wave travelling through the whole layered structure above the mine. In addition to the standard equipment required for the refraction and MASW experiments, this test only involved the need for a radio triggering system to cover the long distances between the shot points and the recording unit located outside the entrance of the mine.

\section{ACQUISITIONS}

The road segment shown in Fig. 1 was investigated by moving the seismic spread three times (triangles, circles, and rhombs in Fig. 1). The spread consists of 24 geophones $(4.5 \mathrm{~Hz})$ with a spacing of $5 \mathrm{~m}$ and each spread was overlapping the neighboring spread by two geophones. Geophones were planted into the narrow strip of hard soil bordering the road. The acquisitions were designed to collect reciprocal refraction data and MASW data simultaneously. Nine shots were fired for each spread, with off-end shots placed at 15 and $45 \mathrm{~m}$ distance from the closer geophone. The team was prepared to use a seismic gun but after a few preliminary shots with an $8 \mathrm{~kg}$ hammer it was decided to accomplish the full work with this source. As a matter of fact, the hammer data were quite good (Fig. 2), a satisfactory SNR was also obtained with the longest experiments (i.e., maximum offset of $160 \mathrm{~m}$ ) and the use of this source was more convenient and faster than the seismic gun. Each shot was repeated twice or more but, generally, the data were not stacked; rather, the best records were selected.

For the transillumination experiments, two $4.5 \mathrm{~Hz}$ geophones were mounted on heavy metal cylinders and were positioned at a distance of $5 \mathrm{~m}$ inside the gallery of the uppermost level of the mine (Fig. 3). The top view of the transillumination geometry is shown in Fig. 7: the two geophones are indicated by the triangles while black stars indicate the 10 shot locations dis- 


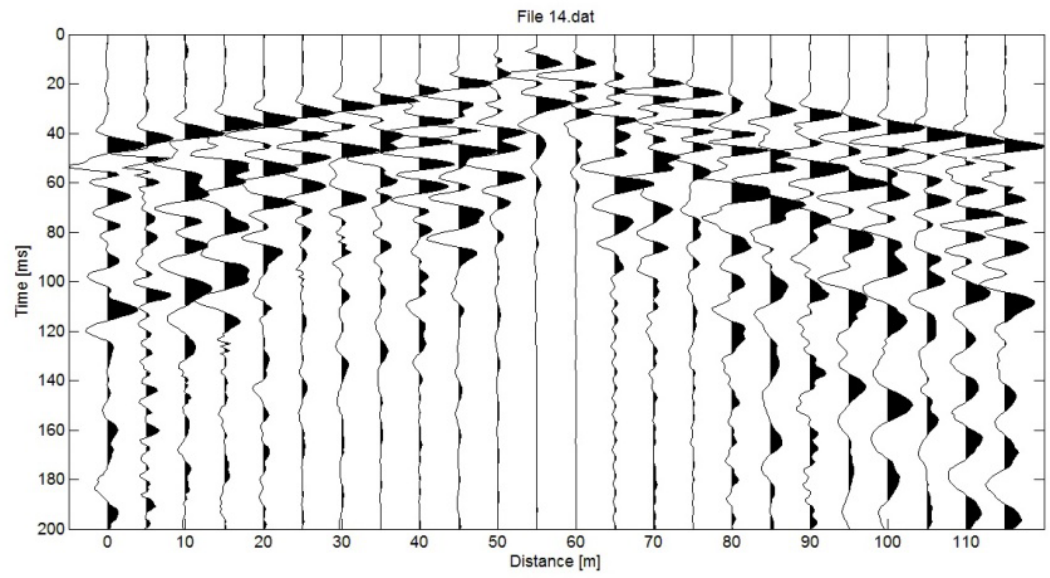

Fig. 2. Example of raw data (windowed at $200 \mathrm{~ms}$ ) generated with the hammer source. Note the good SNR level (traces are normalized to their maxima).

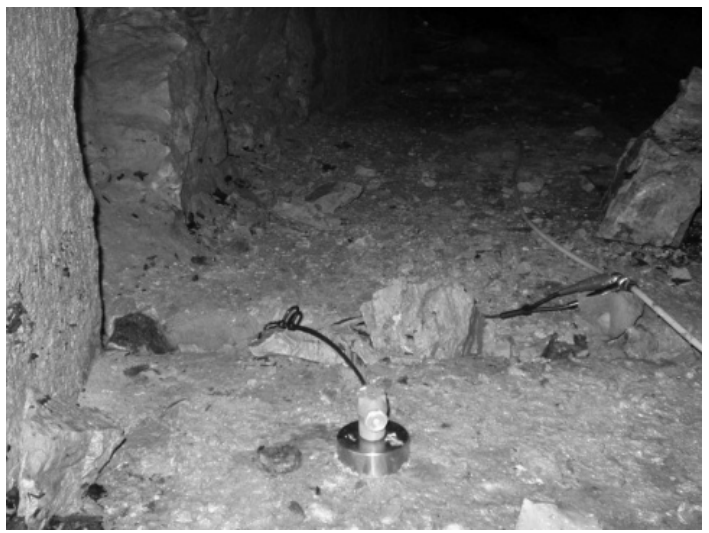

Fig. 3. Deployment of the geophone at the entrance of the mine gallery for the transillumination experiment. The geophone is mounted on a heavy metal cylinder. The cable enters the gallery to reach another geophone at a distance of $5 \mathrm{~m}$.

tributed along the road. Again, the $8 \mathrm{~kg}$ hammer was used and each shot was repeated twice or more to select the best record. The gallery level is about $50 \mathrm{~m}$ below the road elevation. As a result, the range of the 10 shotgeophone distances is between 96 and $227 \mathrm{~m}$. The quality of the data was good enough to perform a reliable first arrival picking up to a distance of $156 \mathrm{~m}$. To carry out the experiments, the seismograph was located outside the entrance of the mine gallery and a radio triggering system was used to synchronize the recording unit with the source. 


\section{RESULTS}

\subsection{Refraction seismics}

Refraction data were inverted by applying the generalized reciprocal method (Palmer 1980, 1981) with a Matlab code developed at Politecnico di Milano. Velocity changes on traveltime curves were rather clear and revealed a 2-layer structure. Figure 4 shows the final model gained by joining the results obtained with the three seismic spreads. Dotted boxes along the road are the $P$-wave velocity estimates in $\mathrm{m} / \mathrm{s}$ obtained for the first layer, while solid boxes are the velocity estimates obtained for the second layer. The thickness of the first layer approximately varies between 7 and $14 \mathrm{~m}$ with the highest value in the area that was affected by the collapse occurred in 1958. By analyzing the velocity in the first layer we can distinguish two zones. West of the parking lot built after the 1958 sinkhole, the first layer velocity is higher, between 1250 and $1400 \mathrm{~m} / \mathrm{s}$. On the right side of the parking lot, the velocity is lower, between 750 and $1000 \mathrm{~m} / \mathrm{s}$. The abrupt variation of the first layer velocity across the parking lot may be most probably due to change in subsurface mechanical parameters because of the collapse and following restoration works. A similar behavior is also observed in the velocity of the second layer.

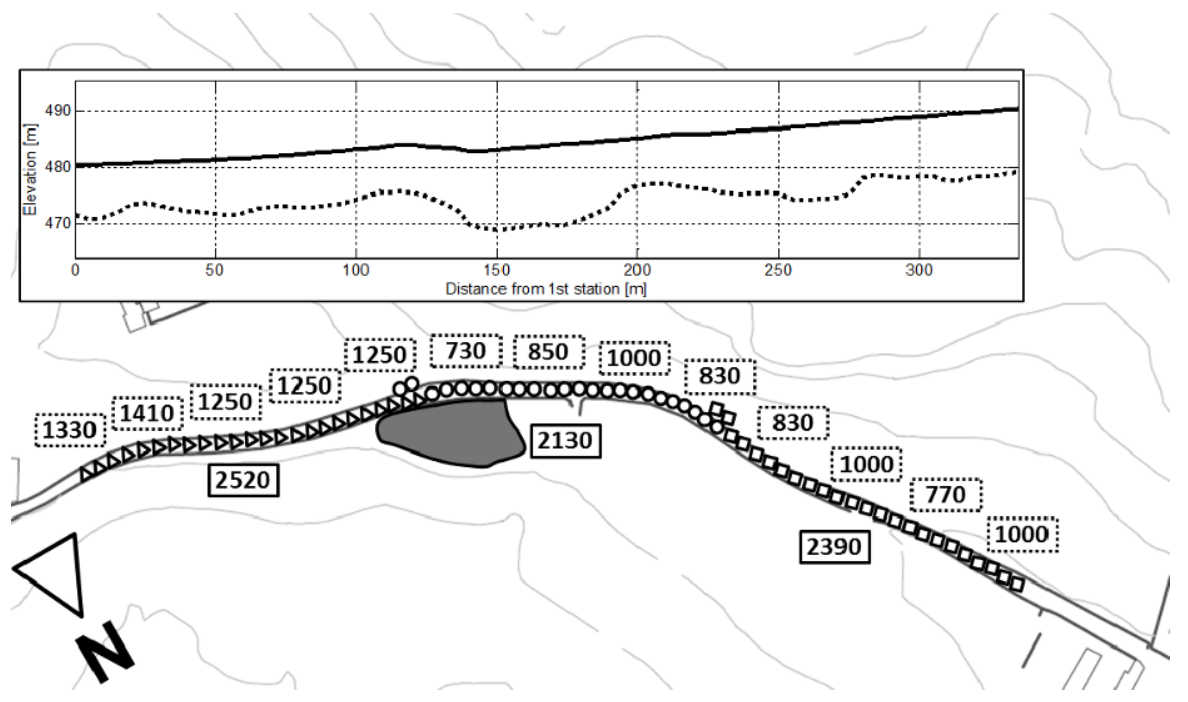

Fig. 4. Results of refraction seismics. The model shows the road topography (solid line) and the interface (dotted line) between the first low velocity layer and the second layer (vertical exaggeration is 2). Dotted and solid boxes indicate $P$-wave velocities in $\mathrm{m} / \mathrm{s}$ in the first layer and in the second layer, respectively. Shaded area is the parking lot. 


\subsection{Multichannel analysis of surface waves}

The MASW technique (Park et al. 1999, Socco et al. 2010) has undergone a rapid development during the last decade and it is currently most utilized for seismic zonation as well as geotechnical applications. Acquisition is generally performed in a roll-along fashion and dispersive Rayleigh wave propagation is analyzed to obtain shear velocity profiles. MASW is considered the easiest seismic method since in a seismic experiment usually more than two third of the source energy is converted into surface wave. Nevertheless, the acquisition parameters, such as source offset and roll increment, are to be set properly in order to limit issues associated with near and far field effects (Socco and Strobbia 2004). Moreover, careful understanding of generated dispersion curves is essential in order to avoid misinterpretation due to the presence of higher modes (Socco and Strobbia 2004). Collected data were processed and, after a preliminary analysis, shot collected with a source offset of $15 \mathrm{~m}$ and a full spread $115 \mathrm{~m}$ long were elected as the best ones. Figure 5 depicts raw stacked shots collected along the three spreads and it is very clear how the middle spread is most favorable for the application of the MASW methodology, since the wider the fanning, the greater the dispersion with depth. Western spread presents a similar situation, although with a lower SNR. Limited dispersion along the eastern spread suggests the presence of a more homogeneous and compact material in that area. Generated dispersion images confirmed what has just been said; the middle spread reveals a classical and well defined dispersion trend (velocity inversely proportional to frequency), while western spread is affected by contamination of higher modes and low frequencies seem to display an unstable behavior, probably related to some near-field effect (surface waves do not behave as plane waves at short distance from the source). The dispersion image related to the eastern spread is heavily contaminated by higher modes and any trend cannot be clearly observed. According to the results of dispersion image analysis, dispersion curves are automatically generated only for middle and western spread and, if necessary, manually edited according to the SNR of the picked points and in order to obtain consistent trends of the curves. Frequency ranges of the picked dispersion curves generally span between 8 and nearly $35 \mathrm{~Hz}$. The maximum depth of investigation in about $60 \mathrm{~m}$, according to the common rule of thumb that relates the maximum investigation depth to half of the maximum wavelength (Shtivelman 1999). Shear wave velocity profiles are finally shown in Fig. 6; results were obtained through a leastsquare iterative procedure and imposing a 10-layer model. MASW velocity profiles are in agreement with refraction seismics findings, since at nearly $11-12 \mathrm{~m}$, a velocity increase is observed. A velocity inversion reveals the presence of a third layer, $10-20 \mathrm{~m}$ thick, that was obviously not detected by 
refraction seismic analysis. A faster fourth layer is then observed, probably immediately above of the uppermost gallery level.



Fig. 5. Raw stacked data collected with a $15 \mathrm{~m}$ source offset. Middle and western spreads present a wider fanning of the acquired signals, while eastern spread is probably placed above a more homogeneous and compact material.

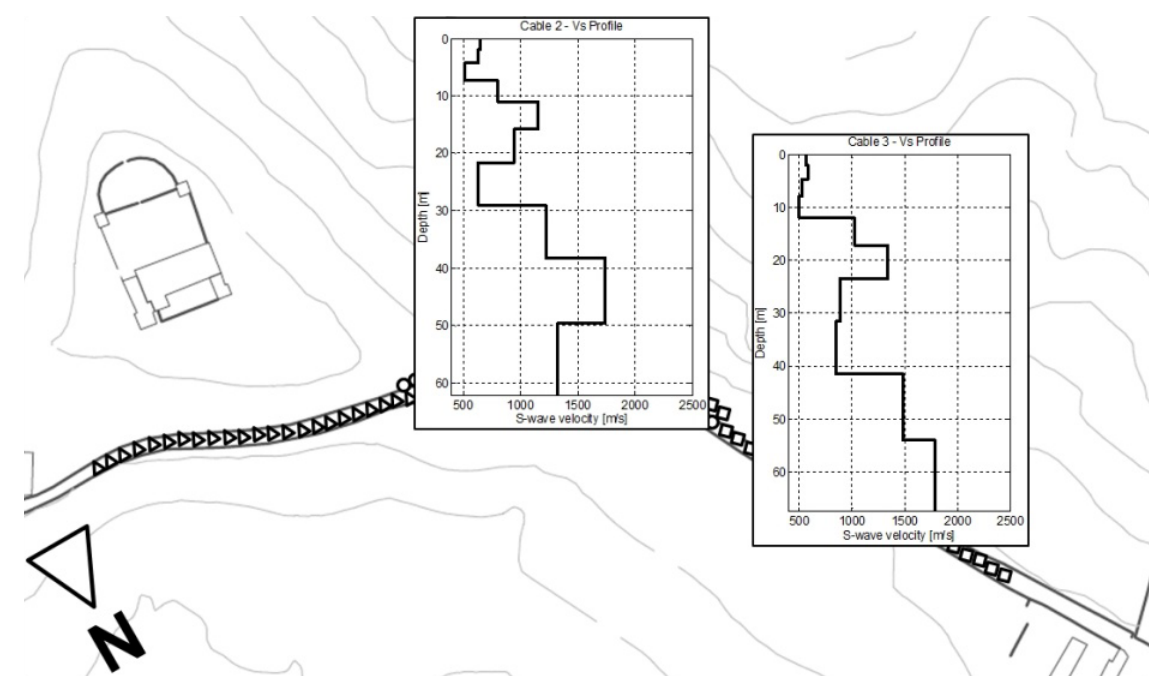

Fig. 6. Shear wave velocity profiles related to middle and western spread. Results are in agreement with refraction seismics and a velocity inversion is identified between the 2 nd and the 4 th layer. 


\subsection{Transillumination test}

In principle, first arrivals picked on transillumination data can only give an estimate of the average velocity of the $P$-wave through the whole rock structure above the upper gallery of the mine. However, the model derived from refraction seismics (Fig. 4) can be used to remove the delay introduced by the low velocity layer and to compute the distance to the geophones from the base of the first layer below each source location. As a result, the average velocity can be attributed to the consolidated layer. Besides, since the source-receiver trajectory is sub-horizontal, the $P$-wave will be refracted generating a head-wave that mainly runs into the highest velocity layer within the rock structure that covers the mine.

Figure 7 summarizes the results. Data from the three farthest shots were not reliable enough to be reported. On the whole, the high velocities obtained indicate the presence of a compact and elastic material. Comparing these values with the velocities of the second layer in the refraction model (Fig. 4) we find a positive feedback that is consistent with the model given by the MASW analysis where the refraction model is downward extended with at least two more layers: a low velocity layer creating a velocity inversion and a high velocity layer faster than the second layer. If this model is true, the transillumination wave is a refracted wave that mainly runs into this high velocity layer and the actual velocity into this layer must be higher than the average values reported in Fig. 7 to compensate for the delay introduced by the low velocity layer detected by the MASW method.

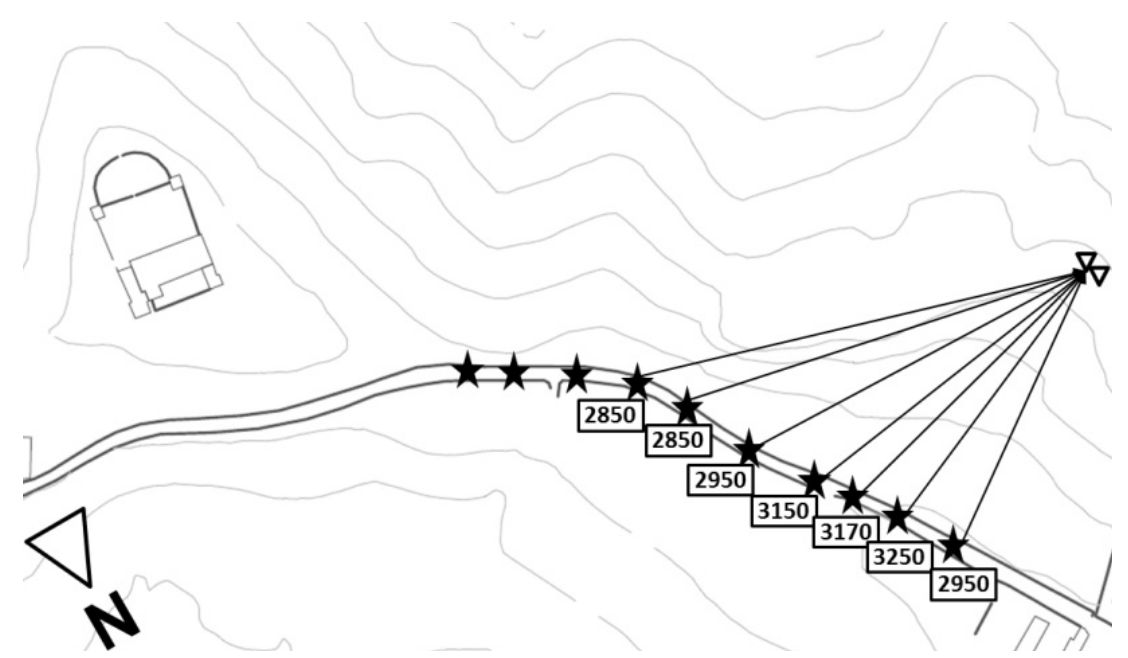

Fig. 7. Average velocities (corrected to remove the delay introduced by the first layer) in $\mathrm{m} / \mathrm{s}$ from the transillumination experiments. 
A small velocity decrease can be noted in Fig. 7 by moving from west to east. This is consistent with the indications derived from both refraction seismics and MASW.

\section{QUALITY FACTOR}

By studying the amplitude decay observed in refraction and transillumination experiments we can obtain some additional information about the quality of the rock structure.

First arrival amplitude reading is quite critical because this parameter is affected by uncontrollable factors such as the geophone coupling. To smooth the influence of local amplitude effects, we averaged all the attenuation data measured along a seismic spread and we determined a single attenuation estimate for each spread. Obviously, direct arrivals and refracted arrivals were separately analyzed to obtain the average attenuation estimates of the first and the second layer, respectively. Before converting the amplitudes into attenuation values, the divergence effect was removed so that the final attenuation estimates can be solely attributed to inelastic absorption and scattering. This was performed differently for the direct waves, where divergence is expected to produce a decay of $20 \mathrm{~dB} /$ decade, and for the refracted waves, where divergence is expected to produce a decay of $40 \mathrm{~dB} /$ decade, provided that distances are sufficiently higher than the critical distance.

Finally, the average attenuation values were converted into average estimates of the rock quality factor according to the following equation

$$
Q=\frac{\pi f}{v \alpha},
$$

where $\alpha$ is the average attenuation, $v$ is the average velocity, and $f$ is the central frequency of the average spectrum $(50 \mathrm{~Hz}$ was used for all the data).

The attenuation analysis was also extended to the transillumination data. Again, amplitudes were preliminarily corrected to compensate for the divergence effect. In this case, the geophone coupling is not changing but the source intensity can vary from shot to shot. Thanks to the multiple records that were collected for each shot location we could evaluate the stability of the source intensity. We found that the amplitude variations related to the source intensity were negligible compared to the amplitude variations associated with absorption. As a result, it was possible to obtain from all the transillumination experiments an average estimate of attenuation that should be mainly attributed to the highest velocity layer of the near surface structure. The resulting attenuation was also converted with the above equation into an average estimate of the quality factor (a velocity of $3000 \mathrm{~m} / \mathrm{s}$ was used for this conversion). 




Fig. 8. Average attenuation values and quality factors from the refraction and the transillumination datasets. Dotted boxes are used for the first layer; solid boxes are used for the second layer and for the transillumination results.

All the results are illustrated in Fig. 8. An average result for each seismic spread is reported (dotted boxes for the first layer, solid boxes for the second layer). Solid boxes close to the mine entrance are the average results derived from the transillumination data and should be attributed to the deepest and highest velocity layer of the rock structure covering the mine.

On the whole, the results are consistent with the previous analysis, i.e., compact and more elastic rock along the first spread on the left (lower attenuation and higher $Q$ values) compared to the following spreads. Transillumination results are associated with the highest velocity layer and they indicate a quality of the rock similar to the quality of the second layer observed along the eastern seismic spread.

\section{CONCLUSIONS}

Both the refraction seismics and MASW observe a first low velocity layer ( $V p$ between 750 and $1400 \mathrm{~m} / \mathrm{s}$ ) with thickness varying between 7 and $14 \mathrm{~m}$, and a second layer with higher velocity ( $V p$ between 2100 and $2500 \mathrm{~m} / \mathrm{s}$ ). The MASW method seems to indicate a thickness for the second layer of about $10 \mathrm{~m}$. Below this layer, the $S$-wave velocity model (Fig. 6) shows a velocity inversion caused by a low velocity layer with thickness approximately varying between 10 and $15 \mathrm{~m}$ and finally a 4th layer with velocity significantly higher than the 2 nd layer. The $P$-wave velocity in the 4 th layer is expected to be higher than $3000 \mathrm{~m} / \mathrm{s}$ since this is the average value measured 
in the layered structure in between the base of the first layer and the level of the uppermost mine gallery by the transillumination experiments.

Geophysical investigations are consistent in defining a physical model (Fig. 9) of the site that can be divided in three main parts, corresponding approximately to the segments of the mountain ridge explored with the three seismic spreads (with an overall length of about $300 \mathrm{~m}$ ). The first $100 \mathrm{~m}$ in the east direction (first spread) shows higher velocities both in the first layer $(1250-1400 \mathrm{~m} / \mathrm{s})$ and in the second layer $(2500 \mathrm{~m} / \mathrm{s})$. These values indicate compact rocks, not severely affected by fractures, as also proved by the absence of dispersive waves, by the low average values of attenuation and by the higher values of the quality factor. The following $100 \mathrm{~m}$ (intermediate spread) shows velocities of about 700-1000 and $2100 \mathrm{~m} / \mathrm{s}$ for the first and the second layer, respectively, while in the last $100 \mathrm{~m}$ (third spread) these velocities increase to higher levels (800-1000 and $2400 \mathrm{~m} / \mathrm{s}$ ), although not as high as in the first spread. Similarly, attenuation levels and quality factors in these two segments (second and third spreads) tend, respectively, to rise and decrease compared to the first spread. On the whole, the segment that is expected to be less elastic and more affected by fractures is the intermediate segment to the west of the parking lot built on the 1958 sinkhole. Here, velocities are lower and average attenuation values are higher.

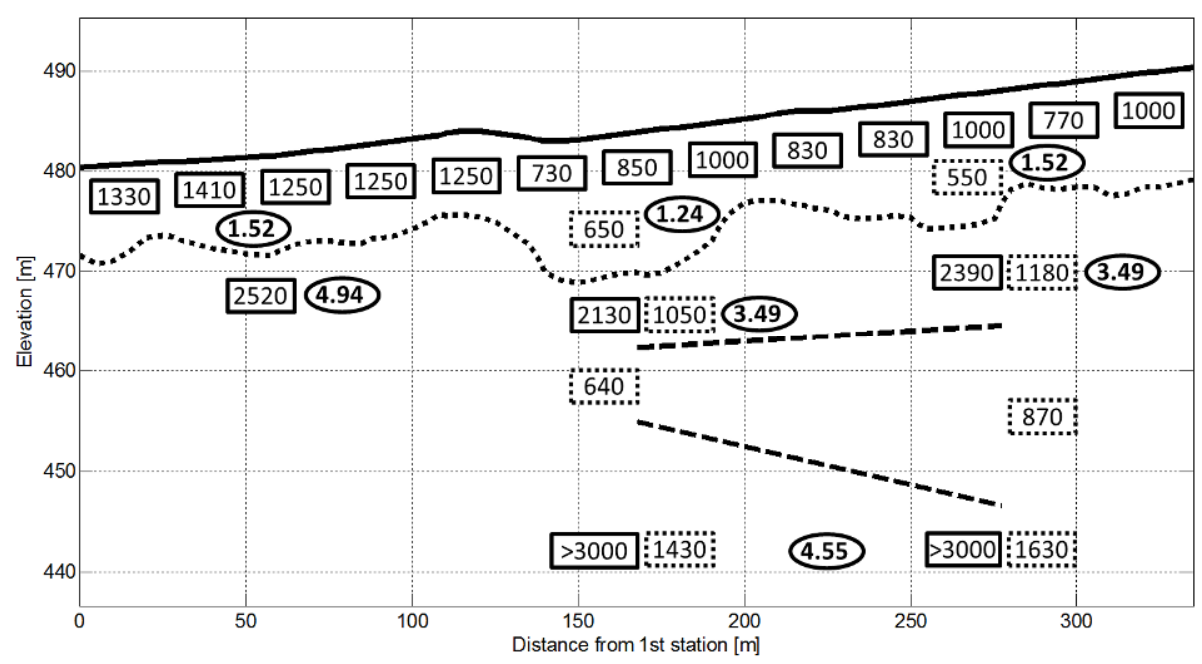

Fig. 9. Results of geophysical investigations. The model shows the road topography (solid line), the interface between first and second layer obtained from $P$-wave refraction (dotted line), and two deeper layers obtained from MASW technique (dashed lines). Solid and dotted boxes indicate $P$ - and $S$-wave velocities, respectively, while ellipses indicate quality factors. 
Acknowledgments. The authors are grateful to Stefano Munda, for his valuable help during the seismic acquisitions, and to Davide Brambilla, who took part to the geological investigations. The comments of an anonymous reviewer helped to improve the quality of the manuscript. The research was funded by Regione Lombardia government.

\section{References}

Palmer, D. (1980), The generalized reciprocal method of seismic refraction interpretation, Society of Exploration Geophysicists, Tulsa, USA.

Palmer, D. (1981), An introduction to the generalized reciprocal method of seismic refraction interpretation, Geophysics 46, 11, 1508-1518, DOI: 10.1190/ 1.1441157.

Park, C.B., R.D. Miller, and J. Xia (1999), Multichannel analysis of surface waves, Geophysics 64, 3, 800-808, DOI: 10.1190/1.1444590.

Shtivelman, V. (1999), Using surface waves for estimating the shear wave velocities in the shallow subsurface onshore and offshore Israel, Eur. J. Environ. Eng. Geophys. 4, 15-35.

Socco, L.V., and C. Strobbia (2004), Surface-wave method for near-surface characterization: a tutorial, Near Surf. Geophys. 2, 4, 165-185, DOI: 10.3997/ 1873-0604.2004015.

Socco, L.V., S. Foti, and D. Boiero (2010), Surface-wave analysis for building nearsurface velocity models - established approaches and new perspectives, Geophysics 75, 5, A83-A102, DOI: 10.1190/1.3479491.

Received 13 April 2012

Received in revised form 4 July 2012

Accepted 31 August 2012 